\title{
Semantic Integration and Sentence Perception ${ }^{1,2}$
}

\author{
SHELDON ROSENBERG \\ Department of Psychology, University of Illinois at Chicago Circle, Chicago, Illinois 60680
}

AND

\author{
ROBERT J. JARVELLA \\ University of Michigan
}

\begin{abstract}
A mixed list of semanticaily well-integrated (SWI) and semantically poorly integrated (SPI) sentences (constructed from associative sentence norms) was presented for shadowing to one group of $S$ s under quiet and to another group under noise ( $-5 \mathrm{db}$ signal-to-noise ratio). The SWI and SPI sentences were balanced for length, number, and stress of syllables, number and type of phones, noun animateness, and word frequency. An incidental-recall task followed one trial of shadowing. As anticipated, shadowing under quiet was virtually perfect for both SWI and SPI sentences, noise reduced shadowing overall and SWI sentences were shadowed better than SPI sentences under noise. Incidental learning of SWI material was enhanced by noise, and noise produced a difference in incidental learning in favor of SWI material.
\end{abstract}

An assumption underlying the present research is that the meaning of a word is represented by (among other things) the linguistic contexts that are part of its dictionary definition and the linguistic contexts that are correlates of the experiences (linguistic and nonlinguistic) commonly associated with the word. Such contexts are referred to here as a word's contextual features. Thus, on this view the predicates in the sentences, The doctor cured the patient and The dog chased the cat, would be considered to be contextual features of their respective subject nouns.

An associative task (Rosenberg \& Koen, 1968) has been used to identify contextual features of nouns functioning as subjects in simple declarative sentences. In this task, a

1 The research reported herein was performed in part pursuant to Contract OEC-3-6-061784-0508 with the U. S. Department of Health, Education, and Welfare, under the provisions of P. L. 85-531, Cooperative Research, and the provisions of Title VI, P. L. 85-864, as amended. The assistance of Mr. David B. Pisoni in obtaining $S \mathrm{~s}$ is acknowledged.

${ }^{2}$ Paper presented at the meeting of the Psychonomic Society, St. Louis, November, 1969. noun is embedded in a sentence frame that contains blanks for the other content words, and the $S \mathrm{~s}$ are instructed to respond to the noun with the words (in the appropriate syntactic classes) that they most frequently associate with it. The data from this task were used to produce norms in which the verb responses for each subject noun are arranged in order of frequency, and beside each verb are arranged the object nouns that occurred with it (also in order of frequency). The verbobject combinations constitute a hierarchy, and it is assumed that the contextual features of a subject noun are the combinations that appear at the top of the hierarchy.

The present study was designed to determine whether contextual features are part of the semantic competence a language user brings to the task of sentence perception. For this purpose, sentences containing contextual features of their subjects-semantically wellintegrated (SWI) sentences-and sentences containing word combinations that occur very infrequently or not at all as responses to their subjects-semantically poorly inte- 
grated (SPI) sentences-were shadowed under conditions of quiet and noise. It was anticipated that because the SPI sentences to be used in the study were not long, anomalous or ungrammatical, and contained mostly high-frequency words, there would be no effect of semantic integration in a quiet environment. Under conditions of noise, however, we did expect to find a difference in shadowing performance in favor of SWI sentences. This expectation was predicated on the assumption that when a portion of a sentence is unintelligible, the remaining portion will be constructed on the basis (mainly) of contextual semantic constraints created by what has already been perceived.

Miller and Isard (1963) had $S$ s shadow a mixed list of meaningful, anomalous and ungrammatical sentences under quiet and noise. Of interest here are the meaningful and anomalous conditions. A number of their meaningful sentences appeared to be SWI (e.g., "A jeweler appraised the glittering diamond earrings."). Their anomalous sentences, however, which were produced supposedly by violating semantic selectional restrictions but which clearly contained instances of syntactic-rule violation as well, were not comparable to the present SPI sentences. In addition, their sentences were generally longer and contained words of lower Thorndike and Lorge (1944) frequency than the sentences used here. Given these conditions, and the demands they must have made upon whatever storage system is involved in shadowing, it is not surprising that Miller and Isard found superior performance for their meaningful sentences under both noise and quiet.

Research on the role of semantic integration in sentence memory (Rosenberg, 1968, 1969; Van Every \& Rosenberg, 1970) has shown that SWI sentences are stored more efficiently (that is, in larger chunks) than SPI sentences, and as a result are recalled better than SPI sentences. A question arises, however, as to whether in the absence of instructions to learn, Ss would store SWI sentences in a manner that would facilitate their recall. This question was evaluated in the present study by administering $S$ s a test of incidental learning immediately after the shadowing task.

\section{METHOD}

Subjects. The $S$ s were 40 paid undergraduate volunteers. All were native speakers of English with no history of hearing disorder. They were assigned to conditions and stimulus presentation orders in rotation, with 20 serving in the quiet condition and 20 in the noise condition.

Materials. The stimuli were 12 SW1 sentences (e.g., The actor played the part; The thief stole the money) and 12 SPI sentences (e.g., The lawyer named the road; The child fixed the sword), all of the same grammatical form. The SWI and SPI sentences were constructed from norms of sequential associative dependency (Rosenberg \& Koen, 1968) and were balanced for number and stress of syllables, number and type of phones, noun animateness, word frequency (Thorndike \& Lorge, 1944), and length. The words in the sentences were mainly AA and A items.

Four balanced (with respect to the location of SWI and SPI sentences) orders of the 24 sentences were used and a master copy was recorded by a male drama student with an Ampex 300 tape recorder. Each sentence was spoken in about $2 \mathrm{sec}$, with 1.5 -sec pauses separating successive sentences. Two experimental versions of each sentence order were prepared by rerecording from the master copy. For both conditions, the speech was recorded onto the first channel of an experimental tape. In addition, for the noise condition only, a correlated masking noise derived from the speech was recorded simultaneously onto the second channel. The speech was first passed through a distortion circuit which flattens the power spectrum while maintaining instantaneous power and reintroducing harmonics of the $F_{0}$ (O'Malley \& Peterson, 1966; O'Malley, 1968). The circuit's output was the masking noise, which permitted the use of a constant signal-tonoise ratio. Quiet and noise versions of 12 practice sentences of intermediate semantic integration and the instructions to $S \mathrm{~s}$ completed the experimental materials.

Apparatus. The materials were played back on an Ampex 351-2 two-track tape recorder. For the noise condition, outputs of the speech and noise channels were mixed to give a constant $-5 \mathrm{db}$ signal-to-noise ratio. This level was found in pre-experimental testing to permit about $50 \%$ word recognition for SWI and SPI sentences combined. For both conditions, all materials were presented binaurally through TDH39 earphones at approximately $85 \mathrm{db}$ SPL re .0002 
dyne $/ \mathrm{cm}^{2}$. The $S$ s were tested individually in an audiometric room.

Procedure. The $S$ s were told that the purpose of the experiment was to discover how people process sentences they hear, that they would hear a tape-recorded list of sentences, and that their task was to repeat each sentence immediately as soon as they heard it and before the next sentence was presented. The $S$ s were encouraged to guess at items they were unsure of. The $S$ s in the noise condition were told that the sentences would be masked. Practice trials listening to and immediately repating the 12 practice sentences followed. All $S$ s were then given the shadowing instructions again and finally were presented with the experimental sentences. Responses were scored by $E$ during the experiment and taped for later rechecking.

Immediately after presentation of the experimental sentences, $S$ s were told for the first time that they would be asked to recall the sentences from the list they had just heard. They were asked to write down all complete and partial sentences they could recall, and were encouraged to guess at items they were unsure of. The $S$ s were given writing materials, and were permitted up to $5 \mathrm{~min}$ to complete the incidental learning task. The interval between the end of shadowing and the beginning of writing was $60 \mathrm{sec}$.

\section{RESULTS}

The shadowing data were scored for the number of complete sentences and content words (nouns and verbs) perceived correctly, as well as for the proportion of instances in which a subject noun (SN) that had been perceived correctly was accompanied by its predicate $(P)$. This last measure was dictated by the manner in which the norms for the SWI and SPI sentences had been produced, and was used as an index of the tendency to perc zive sentences in their entirety.

The means for these measures for the various experimental conditions can be found in Table 1, where it can be seen that $(a)$ shadowing under quiet was virtually perfect for both levels of semantic integration, $(b)$ noise reduced intelligibility appreciably for both SWI and SPI sentences and that $(c)$ shadowing under noise was more successful for SWI sentences. Under noise, $t(19)=12.48$, $p<.001$ for complete sentences; $t(19)=11.79$, $p<.001$, for content words; and $t(19)=12.18$, $p<.001$, for proportion $\mathrm{SN}+\mathrm{P}$. Thus, it is
TABLE 1

Means for Various Measures of Perception

\begin{tabular}{lrrrr}
\hline & \multicolumn{4}{c}{ Group } \\
\cline { 2 - 5 } & \multicolumn{2}{c}{ Quiet } & \multicolumn{2}{c}{ Noise } \\
\hline Measure & SWI & \multicolumn{1}{c}{ SPI } & \multicolumn{1}{c}{ SWI } & \multicolumn{1}{c}{ SPI } \\
Sentences & 11.80 & 11.85 & 4.80 & 1.00 \\
Words & 35.80 & 35.85 & 21.50 & 11.60 \\
SN $+\mathbf{P}$ & .98 & .99 & .77 & .24 \\
\hline
\end{tabular}

clear from these results that semantic integration influences sentence perception under conditions that reduce intelligibility.

An analysis of shadowing accuracy in terms of the position of the content words (that is, subject noun, verb, object noun) was also carried out. Performance under quiet was essentially perfect, of course, but under noise, shadowing accuracy increased linearly from the first to the third position for the words from SWI sentences and increased from the first to the second position and decreased thereafter for the words from SPI sentences. For SWI items, the means for Positions 1, 2, and 3 , respectively, were $6.25,7.15$, and 8.10 , while for SPI items these means were 3.75 , 4.50 , and 3.40. A within- $S$ s analysis of variance for the noise data revealed significant effects of semantic integration, $F(1,19)=$ $123.21, p<.001$; and position, $F(2,38)=5.64$, $p<.01$; and a significant interaction between the two, $F(2,38)=10.44, p<.001$. The significant interaction is due evidently to the fact that performance in relation to position increased continuously for SWI items but fell off after Position 2 for SPI items. An analysis of the simple within effects, with $p=.05$ (Lindquist, 1953), revealed a significant difference between adjacent positions in SWI sentences, but in SPI sentences only the difference between Positions 2 and 3 was significant.

The measure of incidental learning was the proportion of perceived content words that were recalled correctly. An analysis based on complete sentences was not possible due to the 
TABLE 2

Means for Absolute and Proportional Recall of WORDS FROM SWI AND SPI SENTENCES

\begin{tabular}{lrrrr}
\hline & \multicolumn{4}{c}{ Group } \\
\cline { 2 - 5 } & \multicolumn{2}{c}{ Quiet } & & \multicolumn{2}{c}{ Noise } \\
\hline Measure & SWI & SPI & \multicolumn{1}{c}{ SWI } & SPI \\
Absolute recall & 6.65 & 8.45 & 9.50 & 2.65 \\
Proportional recall & .19 & .24 & .45 & .21 \\
\hline
\end{tabular}

fact that seven $S$ s from the noise group failed to perceive any complete SPI sentences. Table 2 contains the means for the incidental learning measure along with the means for absolute recall. It is interesting to note there that not only were more words recalled under noise from SWI sentences than from SPI sentences, but the proportion of perceived items recalled was also higher. Thus, even though $S$ s under noise were "exposed" to fewer SPI items than SWI items (Table 1), they recalled proportionally less of this material. More SPI items were recalled under quiet than under noise, but the proportions differed little. However, for SWI items, both absolute and proportional recall were best under noise. Given that $S$ s were exposed to more SWI items under quiet than under noise (Table 1), one would have anticipated a higher level of absolute recall for SWI items under quiet than was actually observed. It is to be noted also that under quiet, recall of SPI items was superior to recall of SWI items.

Analysis of variance of the results for proportional recall, with noise level as a between$S$ s variable and semantic integration as a within- $S$ s variable, revealed significant main effects for both variables as well as a significant interaction. For noise level, $F(1,38)=18.19$, $p<.001$; for semantic integration, $F(1,38)=$ $11.96, p<.001$; and for interaction, $F(1,38)$ $=28.60, p<.001$. The significant overall effect of noise level is clearly the result of the large difference between noise and quiet for SWI items, while the significant overall effect of semantic integration is obviously the result of the large difference between SWI and SPI items for the noise condition. The difference noted earlier between SWI and SPI items under quiet was not found to be significant.

Taken together, these results suggest strongly that incidental learning of SWI items is enhanced by noise, and that noise produces a difference in incidental learning in favor of items from SWI sentences.

An analysis of the results for proportional recall in terms of position can be found in Table 3, where it can be seen that regardless of differences in semantic integration and noise

TABLE 3

Means for Proportional Recall of Words as a Function of Position

\begin{tabular}{lccc}
\hline & \multicolumn{3}{c}{ Position } \\
\hline Group & One & Two & Three \\
SWI-Quiet & .23 & .18 & .15 \\
SPI-Quiet & .28 & .19 & .24 \\
SWI-Noise & .51 & .41 & .43 \\
SPI-Noise & .32 & .12 & .23 \\
Mean & .33 & .23 & .26 \\
\hline
\end{tabular}

level, performance on words from Position 1 was superior to performance on words from Positions 2 and 3 . In addition, with the exception of the SWI condition under quiet, proportional recall increased somewhat from Position 2 to Position 3. An analysis of variance, with noise level as a between-Ss variable and semantic integration and position as within-Ss variables, revealed a significant main effect for position, $F(2,76)=13.87, p<.001$, but no significant interactions between position and the other variables. The analysis for noise level and semantic integration, and their interaction, of course, produced results identical to those for proportional word recall summed over positions. The effect of position, then, appears to be a uniform one.

\section{Discussion}

It seems reasonable to conclude from these results that when intelligibility is reduced by noise, $S \mathrm{~s}$ will utilize the semantic information 
in what have beeen termed "contextual features" to facilitate performance in a sentence perception task. This conclusion is supported not only by the results for sentences and words, but by the results for the subject-predicate dependency measure as well.

As anticipated, there was no effect of semantic integration when sentences were shadowed without noise. Indeed, under this condition, performance was virtually perfect for both SWI and SPI sentences. This finding raises serious questions about the generality of the results of Miller and Isard (1963) for shadowing under quiet, questions that can only be answered, however, by manipulation of such variables as sentence length, word frequency, semantic selectional restrictions, and syntactic structure. Such questions aside, it can be claimed that an advantage of the present technique for manipulating semantic constraints within sentences is that it does not result in violations of semantic selectional restrictions or syntactic rules.

The effect of word position on shadowing accuracy under noise for SWI sentences observed here suggests that high semantic integration may make an increasing number of contextual cues available to the $S$ as he proceeds through a sentence. The observation that in the absence of high semantic integration intelligibility scores drop off at the end of the sentence suggests that the masking effect of noise may increase to a certain extent if not offset by strong contextual cues. Martin (1968) has reported a similar set of findings for meaningful and anomalous sentences selected from Miller and Isard (1963). As pointed out earlier, a number of Miller and Isard's meaningful sentences appear to be SWI. In Martin's study, shadowing accuracy under noise increased as a function of word position for meaningful sentences but decreased for anomalous sentences. However, the fact that scrambled sentences behaved in a manner similar to the meaningful sentences as far as the effect of word position is concerned, is not consistent with the suggestion that the masking effect of noise may increase within a sentence when semantic constraints are weak, and underscores the need for additional research on this problem. It should be pointed out also that position is a variable that is confounded with grammatical factors in the present study and in Martin's.

The very high level of accuracy of shadowing under quiet with the materials of the present study would appear to indicate that this task was performed with minimal attention to the input and its meaning. The difficulty of shadowing under noise, on the other hand, suggests strongly that this task necessitated a high level of attention to the input and its meaning. From the standpoint of storage and retrieval, increased attention to the input and its meaning is likely to produce more efficient coding, that is, a coding of the input in terms of semantic relationships. It is not surprising, then, that $S$ s in the quiet group did not evidence a sensitivity to semantic integration on the incidental learning task, while $S$ s in the noise group did.

With regard to the effect of position on word recall, what is interesting is the uniformity that was observed. Regardless of differences in semantic integration and noise level, the probability of recall of perceived words was higher for the first word in the sentences than it was for the second and third words. And this in spite of differences between some of the conditions in the wordposition function during shadowing. Unfortunately, with the present design it is impossible to determine whether the overall recall results for word position are related to differences in position or to differences in some more abstract variable such as grammatical function or grammatical class.

The results of a study by Postman and Adams (1960) of intentional and incidental learning of passages varying in contextual constraint are relevant here. These authors report (among other things) that while recall and clustering (chunking) are influenced by order of approximation to English 
under both intentional and incidental learning, the effects are greater under instructions to learn. These findings, and the results of related studies, are interpreted by Postman (1964) to mean that incidental learners emit fewer differential responses to the stimulus materials than intentional learners. Furthermore, on this view, any variable that increases differential responding to the stimulus materials should increase learning. The results of the present investigation indicate that noise would qualify as such a variable.

Thus, on Postman's view, we can speculate that the introduction of instructions to learn under quiet would increase the probability of recall of perceived SWI and SPI items, but that the effect would be greater for SWI than for SPI items. However, because of the difficulty of shadowing under noise, it is questionable whether instructions to learn there would increase recall probability much beyond what was observed in the present study. If this is the case, then intentional recall, at least as regards SPI items, might be adversely affected by noise.

Data relevant to this view come from research (Dallett, 1964; Murdock, 1967; Rabbitt, 1968) on intentional short-term memory for such items as digits and words presented under quiet and noise. This work indicates that items perceived correctly under noise are not recalled as well as items perceived correctly under quiet. Such factors as reduced coding efficiency, interference with rehearsal and the pre-empting of part of short-term. memory capacity by recognition activities have been invoked to explain these findings, but it is not yet clear what precisely is responsible for the phenomenon. Be this as it may, the present results suggest that the adverse effect of noise on recall may be limited to SPI items presented under instructions to learn.

\section{REFERENCES}

DALleTt, K. M. Intelligibility and short-term memory in the repetition of digit strings. Journal of Speech and Hearing Research, 1964, 7, 362-368.
LINDQUIST, E. F. Design and analysis of experiments in psychology and education. Boston: Houghton Mifflin, 1953.

Martin, J. G. Temporal word spacing and the perception of ordinary, anomalous, and scrambled strings. Journal of Verbal Learning and Verbal Behavior, 1968, 7, 154-157.

MILLER, G. A., \& IsARD, S. Some perceptual consequences of linguistic rules. Journal of Verbal Learning and Verbal Behavior, 1963, 2, 217-228.

Murdock, B. B., JR. The effects of noise and delayed auditory feedback on short-term memory. Journal of Verbal Learning and Verbal Behavior, 1967, 6, 737-743.

O'Malley, M. A. H. The experimental study of some linguistic and philosophical analyses of English. Unpublished doctoral dissertation, University of Michigan, 1968.

O'Malley, M. A. H., \& Peterson, G. E. An experimental method for prosodic analysis. Phonetica, 1966, 15, 1-13.

Postman, L. Short-term memory and incidental learning. In A. W. Melton (Ed.), Categories of human learning. New York: Academic Press, 1964, Pp. 145-201.

Postman, L., \& Adams, P. A. Studies in incidental learning: VIII. The effects of contextual determination. Journal of Experimental Psychology, 1960, 59, 153-164.

RABBIT, P. M. A. Channel-capacity, intelligibility and immediate memory. Quarterly Journal of Experimental Psychology, 1968, 20, 241-248.

RoSenberG, S. Association and phrase structure in sentence recall. Journal of Verbal Learning and Verbal Behavior, 1968, 7, 1077-1081.

RosenberG, S. The recall of verbal material accompanying semantically well-integrated and semantically poorly integrated sentences. Journal of Verbal Learning and Verbal Behavior, 1969, 8, 732--736.

RosenberG, S., \& Koen, M. J. Norms of sequential associative dependencies in active declarative sentences. In J. C. Catford (Ed.), Studies in language and language behavior, supplement to progress report VI. Ann Arbor: University of Michigan, Center for Research on Language and Language Behavior, U.S.O.E. Contract OEC-3-6061784-0508, February 1, 1968.

THORNDIKE, E. L., \& LoRGE, I. The teacher's word book of 30,000 words. New York: Teachers College, Columbia University, Bureau of Publications, 1944.

VAN Every, H., \& RosenberG, S. Semantics, phrase structure and age as variables in sentence recall. Child Development, 1970, in press.

(Received May 5, 1970) 